\title{
Computation and the Natural World
}

\author{
C. T. A. Schmidt
}

For the past sixty years various philosophers and scientists have claimed that computational accounts are the proper kind of accounts for explaining some complex phenomena of the natural world. Computational theories have been offered as a basis for understanding minds, brains, language, cognition, consciousness, intentionality, life, and evolution. The papers in this volume, both pro and con, assess in what ways such computational accounts will likely succeed or fail. 